\title{
Pixel/Voxel-Based Machine Learning (PML) and Big Data in Medical Imaging: Detection and Regulation
}

\author{
*Sunil Aggarwal, Sanju A and Somya Aggarwal \\ Master Black Belt Lean Six Sigma, USA
}

Submission: November 24, 2016; Published: December 08, 2016

"Corresponding author: Sunil Aggarwal, Master Black Belt Lean Six Sigma, USA.

\begin{abstract}
Medical imaging has always been an essential component of patient management. The pixel/voxel- based machine learning (PML) in medical imaging is gaining momentum as a computer aided diagnostic (CAD) tool if it can achieve better results than radiologists, in terms of detection and bringing down costs. CAD detection of breast cancer in mammograms is one area that has proved to be smarter, but again most radiologists are suspicious of eventual outcome. Machine learning using pixel/voxel values in medical images has shown emergence as a better diagnostic tools than the segmentation or feature based input. Pixel/Voxel based calculations avoid error that is inherent to segmentation based information. Once regulatory information is used as a metadata, new opportunities of machine learning in regulatory compliance would emerge and the regulatory framework would be based on the regulatory requirements and minimizing costs in regulatory compliance. Eventually, the potential for risk analysis may become automated.
\end{abstract}

Keywords: Computer aided diagnosis; Pixel; Voxel; Machine learning; Medical imaging; Regulatory

\section{Introduction}

Machine learning uses algorithms derived from empirical data and make informed decisions based on learning from prior. Analysis of medical images, segmentation, CAD, and other medical image involving methods such as image fusion or image guided therapy are all becoming possible by machine learning. Pixel and voxel metrics define the prior learning experience of the machine learning from previous examples and then un turn guides these machines to diagnose MRI, CT, PET-CT (positron emission tomography hybrid with $\mathrm{CT}$ ) would require machine learning based on new algorithms and data. Pixel/voxel metrics would generate more diagnostic data than information obtained from segmentation of an organ, as errors can be avoided by pixel/voxel based machine learning.

\section{a)What is pixel, voxel and an image?}

A pixel, also known as picture element (pics for pictures, el for element), is the most basic and smallest unit of a digital image that could be displayed and controlled. It is a point sample and only exists at a point [1,2]. Pixel is thus a sample of its image. When there is a color image, there may be a combination of three colors, red, green and blue, and three such samples, making up a pixel. An image is, on the other hand, is a collection of many pixels. In a CT scan image, a pixel is a two dimensional sample matrix and is displayed by the attenuating tissue in the form of a grey scale based on Hounsfield unit [2]. Voxel, on the other hand, represents a volume unit and is a three dimensional unit. This means that the CT image is analyzed through many pixels and their brightness depends on the X-Ray absorption or attenuation capacity of voxels [2].

\section{Pml Based Medical Imaging}

PML based machine learning incorporates the use of intelligent systems that use prior learning experiences derived from big data. Anatomical structures and pathological states are identified from normal state through pattern recognition and adjustment of its features in its algorithm. Data helps to identify abnormal from normal anatomy based on pixel and voxel characterization. In medical imaging, and in segmentation of pathology, use of lean methods to expedite diagnosis can save time $[3,4]$. This is why it is classified as Pixel/voxel based [5] and when compared to conventional forms of segmentation (Figures $1 \& 2$ ), PML is highly diagnostic in identification and diagnosis with a predictive value of labeling as benign or malignant (Figure $3)$. Proves to be a highly accurate method of segmentation of pathologic anatomy and diseased states, when compared to Segmentation can be performed in a semi-automated or 


\section{Cancer Therapy \& Oncology International Journal}

manual representation detailing most external as subcutaneous adipose tissue, muscle bundle and the innermost bone [6]. PML is reproducible and thus of desired use with supervised, unsupervised or reinforcement learning. These learning methods could be data and label driven, only data driven and reacting to environment using an algorithm respectively (Figure 4) [7].

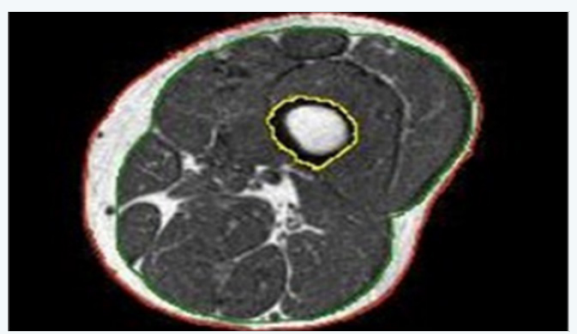

Figure 1: Segmentation of muscle bundle volume outlined in green, bone in yellow and subcutaneous Fat in red.

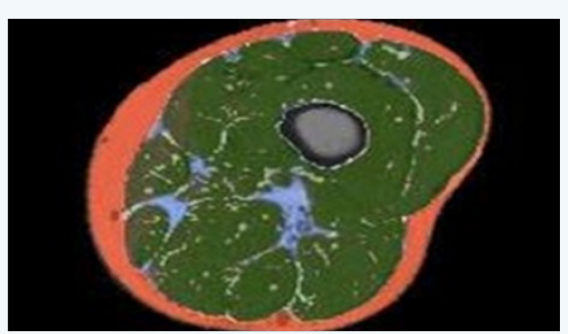

Figure 2: Representation of muscle bundle volume painted in green and subcutaneous adipose tissue in red.

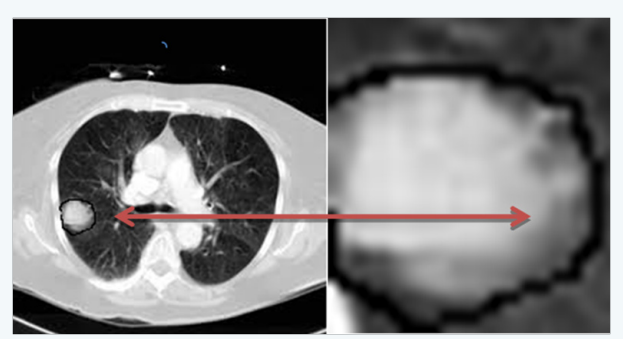

Figure 3: PML: aid in identfication, diagnosis of cancer tumor with classification as malignant or benign.

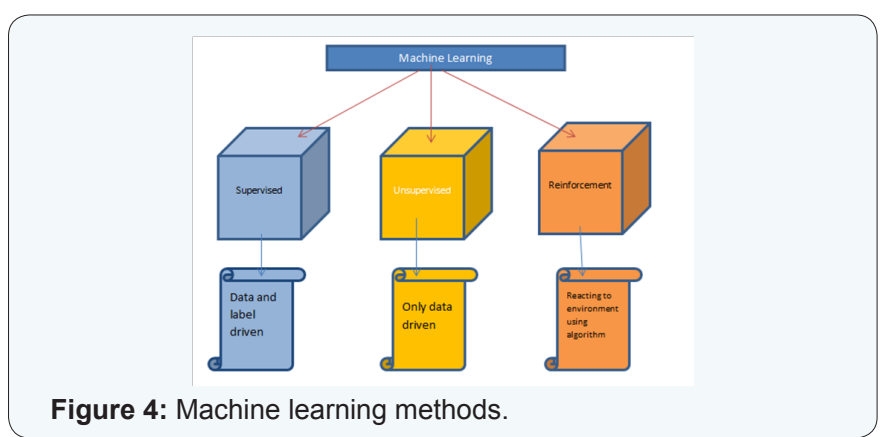

\section{a)Disadvantages of PML}

The most obvious disadvantage, at present, is a lack of informative data that may help to compute further as PML is data driven. It is expensive and can take only small identifier set as features and if there is a wide spread lesion, as in whole of left lung, it is difficult for PML to retrieve all the required information to diagnose. Discontinuous loss functions that are not differentiated are difficult to work with PML. Large amount of data is required to learn from prior experiences for PML and it is not always a must that PML would work in all scenarios [5]. The functional physiological mechanisms or physiologic modeling should correlate to the information received from the machine learning.

\section{b)Regulation of PML}

i. FDA has classified two types of CAD devices: CADe and CADx [8]. CADe devices are "computerized systems that incorporate pattern recognition and data analysis capabilities (i.e., combine values, measurements or features extracted from the patient radiological data) and are intended to identify, mark, highlight or in any other manner direct attention to portions of an image, or aspects of radiology device data, that may reveal abnormalities during interpretation of patient radiology images or patient radiology device data by the intended user." CADx devices are "computerized systems intended to provide information beyond identifying, marking, highlighting or in any other manner directing attention to portions of an image, or aspects of radiology device data, that may reveal abnormalities during interpretation of patient radiology images or patient radiology device data by the clinician"[8]. In 2012, FDA documented that CADe devices could follow the $510(\mathrm{k})$ process for their clearance and CADx would follow premarket approval.

Mammography CAD has still not been classified as CADe device through FDA and this implies that $510(\mathrm{k})$ route does not apply here. PML medical devices would also have to follow premarket approval route as no predicate devices would exist for FAD to allow a $510(\mathrm{k})$ route for these. No substantially equivalence would exist for these algorithms. This would mean a tougher route of FDA clearance and that PML medical devices may take a while to come to the market, even if the PML device companies start early on with FDA. However, CAD devices could offer another opinion for screening cancers, like lung cancers [9].

\section{Conclusion}

Machine learning requires an algorithm and a data. Data of patients and within a patient caries and algorithms may not be a feasible option in diverse use of machine learning in medical sciences, but certainly it has a ground of support for medical imaging. The brightness characterization of a pixel and its volume in a picture can give more information than a human eye or diagnose more is justified in some instances but needs more to explore. Cancer diagnosis, prognosis and predictions would become possible through PML $[5,10]$. Regulations for PML medical devices would not be in the very near future, if they follow premarket approval of FDA. PML will revolutionize the medical imaging, once it gets going, although this may happen outside US earlier than one would expect. 


\section{Cancer Therapy \& Oncology International Journal}

\section{References}

1. Alvy Ray Smith (1995) A Sprite Theory of Image Computing. Tech Memo 5, Microsoft, Jul 17.

2. H H Barrett and W Swindell (1981) Radiological Imaging. The Theory of Image Formation, Detection and Processing, I, Academic Press, New York, USA, 438-439.

3. Sunil Aggarwal, Sanju A, Somya Aggarwal (2016) A Study of Lean in Oncology: Reducing Waste and Increasing Value. Canc Therapy \& Oncol Int J 1(3): 555563.

4. Sunil A and Somya A (2016) Lean Six Sigma for Medical Device Excellence and Regulation. Adv Pharmacol Clin Trials 1(2): 000111.

5. Joseph A Cruz and David S Wishart (2006) Applications of Machine Learning in Cancer Prediction and Prognosis. Cancer Informatics 2: 59-77.
6. Somya Aggarwal, Kareem B, Sunil Aggarwal, Jasreen G, Ali Abdulla, et al. (2016) Thigh Muscle Bundle Volume by Magnetic Resonance Muscle Quantification(MRMQ) in Skeletal Muscle Wasting and Relevance of Supine Rest. Canc Therapy \&OncolInt J 1(2): 555560.

7. O Chapelle, B Scholkopf and A Zien Eds (2009) Semi-Supervised Learning (Chapelle, O. et al. (Eds.) [Book reviews] in IEEE. Transactions on Neural Networks 20(3): 542-542.

8. Abe Y, Hanai K, Nakano M, Ohkubo Y, Hasizume T, et al. (2005) A computer-aided diagnosis (CAD) system in lung cancer screening with computed tomography. Anticancer Res 25(1B): 483-488.

9. Kourou K, Exarchos TP, Exarchos KP, Karamouzis MV, Fotiadis DI (2015). Machine learning applications in cancer prognosis and prediction. Compu Structural Biotechnol J 13(8-17).

10. Abbass HA (2002) An evolutionary artificial neural networks approach for breast cancer diagnosis. Artif Intell Med 25(3): 265-281.

Your next submission with JuniperPublishers will reach you the below assets

- Quality Editorial service

- Swift Peer Review

- Reprints availability

- E-prints Service

- Manuscript Podcast for convenient understanding

- Global attainment for your research

- Manuscript accessibility in different formats ( Pdf, E-pub, Full Text, Audio)

- Unceasing customer service

Track the below URL for one-step submission http://juniperpublishers.com/online-submission.php 